\title{
A Simple Method To Improve the Clarity and Rheological Properties of Polymer/Clay Nanocomposites by Using Fractionated Clay Particles
}

\author{
Bani H. Cipriano, ${ }^{\dagger,}{ }^{\ddagger}$ Takashi Kashiwagi, ${ }^{\S}$ Xin Zhang," and Srinivasa R. Raghavan ${ }^{*}, \dagger$
}

Departments of Chemical \& Biomolecular Engineering and Materials Science \& Engineering, University of Maryland, College Park, Maryland 20742-2111, and Fire Research Division, National Institute of Standards \& Technology,

Gaithersburg, Maryland 20899

\begin{abstract}
We present a simple method to improve the transparency and rheological properties of polystyrene/organoclay nanocomposites. Such composites are typically prepared using organophilic derivatives of commercial montmorillonite (MMT) clays that contain a wide range of particle sizes. Our approach is to fractionate the native MMT clay to exclude large aggregates and then modify the fractionated clay (FMT) by a surfactant treatment (the resulting organoclay is termed o-FMT). Polystyrene nanocomposites made from the o-FMT are then compared with those made from a commercial organoclay having the same surfactant treatment. The o-FMT-based composites exhibit much better rheological properties (e.g., their elastic moduli at low frequencies are $\sim 10$ times higher) at equivalent mass loadings. Moreover, the o-FMT-based materials also have better transparency: e.g., a $10 \%$ o-FMT sample transmits as much light as a sample with $5 \%$ commercial clay. Transmission electron microscopy confirms that these differences in properties are related to microstructural differences; i.e., large aggregates are generally absent in the o-FMT samples. Clay fractionation may thus be a general strategy to enhance the properties of polymer/clay nanocomposites.
\end{abstract}

KEYWORDS: polymer nanocomposites $\bullet$ organoclay $\bullet$ rheology $\bullet$ transparency

\section{INTRODUCTION}

1 olymer nanocomposites made by mixing thermoplastic polymers with clay particles have been extensively studied over the past decade or so $(1-5)$. The use of the term "nanocomposite" for these materials is indeed appropriate when the clay particles are mostly dispersed as individual platelets, each about $1 \mathrm{~nm}$ thick. When such an "exfoliated" morphology is realized, the mechanical and rheological properties of the nanocomposite are dramatically enhanced compared to that of the pure polymer $(1-3)$. Clay particles can also impart other useful properties to the polymer, such as flame retardancy and barrier properties $(2,5,6)$. The prospect of such property enhancements has stimulated interest in polymer/clay nanocomposites for various applications. Clay particles are desirable because of their low cost, especially compared to more exotic particles such as carbon nanotubes

In practice, the fully exfoliated morphology of polymer/ clay materials remains somewhat of an idealization. Complete exfoliation is often possible only under special manufacturing circumstances, such as by in situ polymerization

* Corresponding author. E-mail: sraghava@eng.umd.edu.

Received for review September 23, 2008 and accepted November 30, 2008

† Department of Chemical \& Biomolecular Engineering, University of Maryland

* Present address: Balchem Corporation, New Hampton, NY 10958.

$\S$ National Institute of Standards \& Technology

" Department of Materials Science \& Engineering, University of Maryland.

DOI: $10.1021 /$ am8000693

๑ 2009 American Chemical Society of monomer/clay mixtures (1-3). In conventional processing of polymers by melt extrusion, compounding, or spinning, the addition of clay particles often leads to a morphology where individual (exfoliated) platelets coexist with stacks of platelets (also called "tactoids") (1-5). In the latter case, the polymer chains are intercalated within the platelet stacks. Stacks of clay platelets have a lower aspect ratio because of the stack thickness and are thus less effective at reinforcing the polymer. However, even such partial exfoliation can significantly improve the properties of the polymer, which may be adequate for many applications. The one property of polymers/plastics that is adversely impacted by the addition of clay is the clarity or transparency (the composites are translucent or opaque at high clay loadings), which can be a problem for applications where transparent plastics are desired (this problem is exacerbated with carbon nanotubes) $(2,3)$. Clearly, true nanoscale dispersion of clay particles would ensure the best clarity while also maximizing the rheological properties.

The above discussion highlights the need for improving the nanoscale dispersion of clay particles in polymer/clay nanocomposites. Current efforts to improve dispersion have focused on the type of surface modification conferred to the clay. It is known that the clay surface must be rendered hydrophobic by surfactant modification in order to ensure good dispersion in most thermoplastic polymers $(1-5)$. Thus, the type of surfactant (one- or two-tail, ammonium or imidazolium headgroup, etc.) and the chain length of the surfactant have been evaluated for their impact on clay 


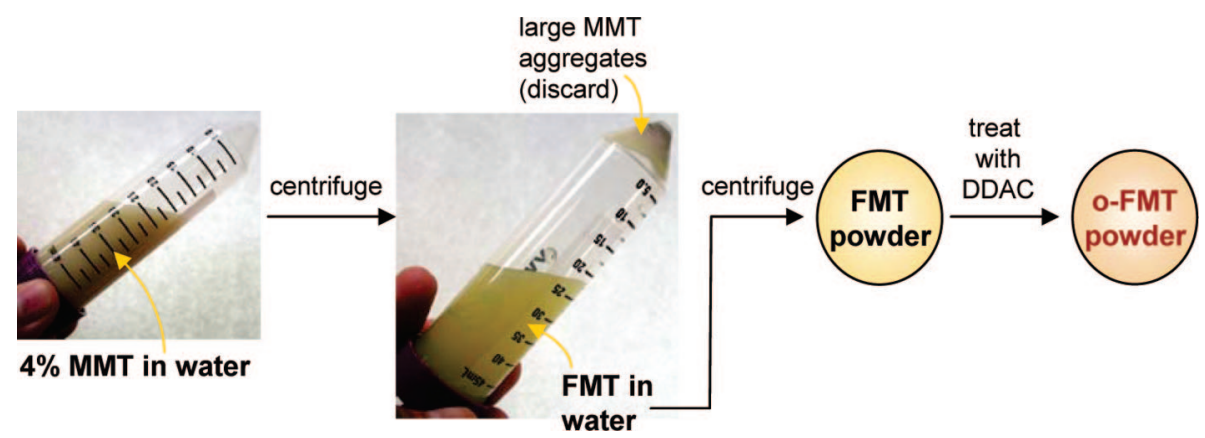

FIGURE 1. Extraction and synthesis of FMT and o-FMT particles. The original MMT dispersion in water is centrifuged to separate out the large aggregates. The supernatant is centrifuged again, and the pellet is FMT. These particles are then functionalized with the cationic surfactant DDAC to yield the o-FMT particles.

dispersion (1-3). For hydrophobic polymers like polystyrene (PS) or polyethylene, a different surface treatment is found to be the most effective at ensuring exfoliation and that is to tether oligomeric chains of the matrix onto the clay surface $(2,3)$. Currently, commercial suppliers, such as Southern Clay Products, provide a variety of "organoclay" particles with different surface treatments of native montmorillonite (MMT). For each grade of organoclay, the manufacturer specifies the surfactant used for the treatment, such as ditallow dimethylammonium chloride (DDAC). Optimizing clay dispersion in a given polymer then becomes a matter of selecting the right grade of organoclay for that polymer.

In this study, we undertook a different approach to improving the properties of polymer/organoclay composites. Our approach arose from the observation that the native MMT (and, thereby, all organoclays prepared from it) is highly polydisperse mixtures of clay particles. Indeed, while MMT is supposed to become fully exfoliated (dispersed as single-nanoscale platelets) in water, aqueous dispersions of MMT are actually quite turbid and reveal large particles/ aggregates (several microns in size) when observed under optical microscopy (see Figure 2). We speculated that such large aggregates were also present in organoclay dispersions and nanocomposites and that in all cases they were undesirable; i.e., they were largely ineffective at reinforcing the matrix. Our approach, therefore, is to remove these large aggregates from an MMT aqueous dispersion using a centrifugation step. The remaining particles constitute the smaller and/or less aggregated fraction of the clay, and we collectively refer to them as fractionated montmorillonite or FMT. We then treat this FMT with DDAC surfactant to obtain its organophilic counterpart (o-FMT). o-FMT is then combined with PS, and the resulting composites are characterized using different techniques. A comparison of the o-FMT composites with those made from a commercial organoclay (also modified by DDAC) shows that the o-FMT materials have significantly better optical and rheological properties.

\section{EXPERIMENTAL SECTION}

Fractionation of MMT. The MMT used was a sodium montmorillonite (Cloisite NA+) from Southern Clay Products. Dispersions of this MMT ( $4 \mathrm{wt} \%$ ) were prepared in distilled-deionized water by stirring continuously using a magnetic stirrer for 3 days. This dispersion, which was a turbid brown liquid (see

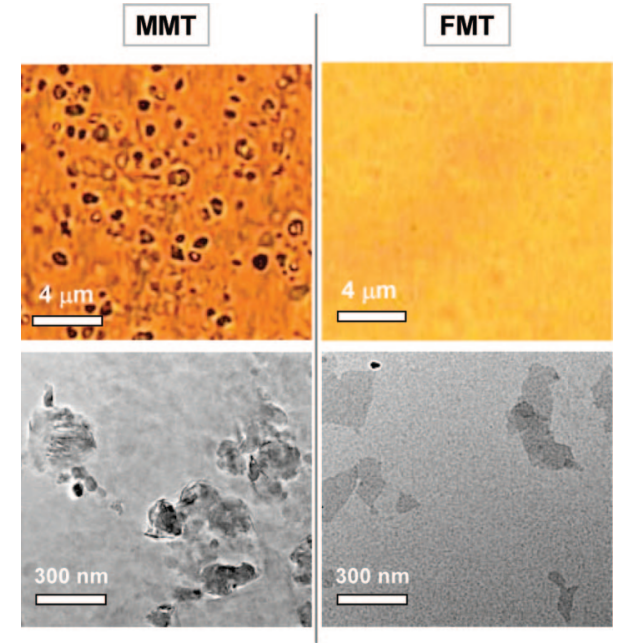

FIGURE 2. Optical (top) and TEM (bottom) images of MMT and FMT clay particles in an aqueous dispersion. The particle concentration was $2 \%$ in both dispersions tested. Large micron-sized aggregates are seen in the case of MMT, while FMT is seen to exist as nanosized clay platelets.

Figure 1), was centrifuged for $1 \mathrm{~h}$ at $3200 \mathrm{~g}$. The precipitate from this centrifugation step contains the largest clay particles and aggregates ( $\sim 20 \%$ of the starting material). The supernatant from this centrifugation was a yellowish fluid, more transparent than the original dispersion (Figure 1). This supernatant was then further centrifuged at $18500 \mathrm{~g}$ for $1 \mathrm{~h}$. The precipitate from this second centrifugation step was collected, while the supernatant was discarded. The clay in the second precipitate is what we will refer to as FMT. For a given amount of starting material, about $50 \%$ was FMT.

Particle Characterization. Dispersions of FMT and MMT in water were characterized by optical microscopy using a Zeiss Axiovert 100 TV inverted microscope with a $20 \times$ objective and by transmission electron microscopy (TEM) using a JEOL JEM$2100 \mathrm{~F}$ microscope. For TEM, a very dilute dispersion of each clay $(0.05 \mathrm{wt} \%)$ was made and a drop of this dispersion was dried on a copper grid.

Synthesis of o-FMT. The FMT from above was diluted to 1.5 wt \% with distilled-deionized water and heated to $65{ }^{\circ} \mathrm{C}$. Simultaneously, DDAC (trade name Arquad, from Fluka) was dissolved in a $450 \mathrm{~mL}$ mixture of 60:40 water/ethanol by heating to $65^{\circ} \mathrm{C}$. The amount of DDAC added to the FMT dispersion corresponded to twice the cation-exchange capacity of the native MMT, known to be 92.6 mequiv per $100 \mathrm{~g}$ of clay. The clay dispersion and DDAC solution were combined and allowed to react overnight with constant stirring. o-FMT was then precipitated, recovered by vacuum filtration, and dried. Excess DDAC from o-FMT was removed by Soxhlet extraction in ethanol for 3 days. o-FMT was then dried prior to use. 
Purification of Commercial Organoclay. We compared o-FMT to a commercial organoclay, Cloisite 15A, also from Southern Clay Products. This product is formed by treatment of the native MMT with DDAC and is known to contain up to $36 \%$ excess of DDAC relative to its cation-exchange capacity (7). The excess DDAC was removed by Soxhlet extraction in ethanol much like with o-FMT. The purified Cloisite 15A was then dried prior to further use.

Thermogravimetric Analysis (TGA) of Organoclays. The organic contents of purified o-FMT and Cloisite 15A were compared by TGA using a Shimadzu TGA-50 instrument. Samples were held at $100{ }^{\circ} \mathrm{C}$ for $15 \mathrm{~min}$ (to eliminate any moisture) followed by a ramp to $800{ }^{\circ} \mathrm{C}$ at a rate of $10^{\circ} \mathrm{C} / \mathrm{min}$. From the mass loss recorded in TGA, the organic content was determined for each set of particles.

Preparation of Nanocomposites. Polymer/clay nanocomposites were prepared by combining o-FMT or the purified Cloisite 15A with a high-molecular-weight PS (Styron 666D, Dow Chemical). The PS had a mass-average molecular weight of $240000 \mathrm{~g} / \mathrm{mol}$ with a polydispersity index of 2.29. PS/clay nanocomposites were prepared by solvent casting. A dispersion of $1 \%$ organoclay in chloroform was prepared by sonication for $5 \mathrm{~min}$, followed by stirring for $2 \mathrm{~h}$. A solution of $10 \mathrm{wt} \%$ PS in chloroform and the organoclay dispersion were combined, and the mixture was stirred for $24 \mathrm{~h}$. Thereafter, this mixture was cast into an aluminum foil pan, and the chloroform evaporated in a hood overnight. The nanocomposite was then further dried and annealed at $160{ }^{\circ} \mathrm{C}$ in a vacuum oven for $48 \mathrm{~h}$.

$\mathrm{X}$-ray diffraction (XRD). XRD spectra on polymer/clay nanocomposites were collected on a Philips diffractometer using $\mathrm{Cu} \mathrm{K} \alpha$ radiation $\left(\lambda=1.54 \AA\right.$ ) with a $2 \theta$ step of $0.04^{\circ}$ and a 3 s count time per step. Samples for XRD were prepared by hot-pressing nanocomposites into 2-mm-thick plates. The stacking distance $d$ of the clay in the nanocomposites was determined from the position of the peak at lowest $2 \theta$ using Bragg's law, $d=\lambda / 2 \sin \theta$

TEM. The microstructures of the polymer/clay nanocomposites were analyzed using a JEOL JEM-2100F TEM operating at $200 \mathrm{kV}$ and equipped with a Gatan CCD camera. Specimens of $60 \mathrm{~nm}$ thickness were prepared by ultramicrotoming (Leica UC6) with a Diatome diamond knife (Hatfield, PA).

Transparency Measurements. The transmittance of composite films was characterized by a Varian Cary 50 UV-vis spectrophotometer (Palo Alto, CA) over wavelengths ranging from 200 to $800 \mathrm{~nm}$. The film thickness was $200 \mu \mathrm{m}$.

Rheology. Samples for rheology were prepared into disks of $25 \mathrm{~mm}$ diameter and $1 \mathrm{~mm}$ thickness by hot-pressing at a temperature of $140{ }^{\circ} \mathrm{C}$. Rheological experiments were performed at $180^{\circ} \mathrm{C}$ on an RDA-III strain-controlled rheometer (TA Instruments) equipped with a convection oven. All experiments were conducted in a nitrogen atmosphere to limit oxidative degradation of the polymer. A parallel-plate geometry $(25 \mathrm{~mm}$ diameter) was used at a gap of $0.9 \mathrm{~mm}$. Measurements were performed under dynamic oscillatory shear. Dynamic frequency sweeps were conducted within the linear viscoelastic regime of the sample, which was determined separately from strain sweep experiments.

\section{RESULTS AND DISCUSSION}

MMT/FMT Dispersions in Water. Figure 1 depicts the extraction of FMT from the original MMT. It is worthwhile to compare the properties of these two sets of clay particles, specifically their dispersions in water, because native clay particles are hydrophilic and are known to form aqueous colloidal dispersions (8-11). As shown in Figure 1, aqueous dispersions of MMT are more turbid compared to those of FMT. Brightfield optical microscopy confirms that the turbid-

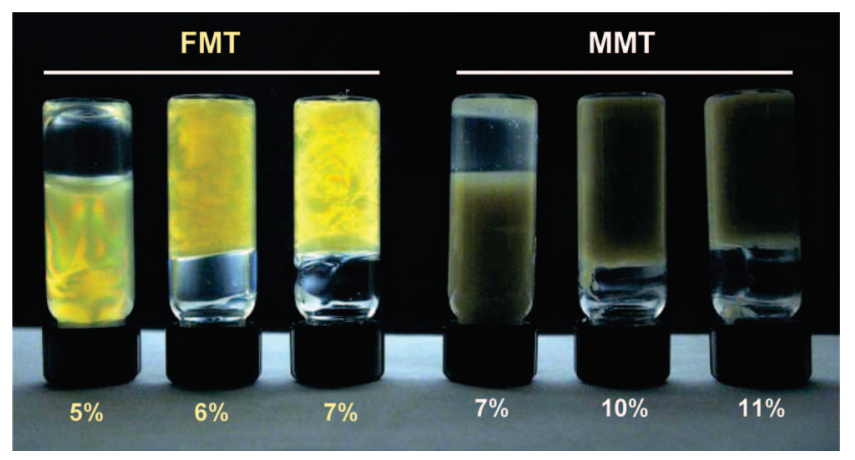

FIGURE 3. Gelling efficiency in water of the original MMT particles (broad distribution) vs the FMT particles (fractionated to remove large aggregates). Samples corresponding to the indicated concentrations are shown in inverted vials and viewed under crossed polarizers. FMT gels water at lower concentrations than MMT, and the FMT gels are birefringent.

ity of MMT is due to the presence of large aggregates (Figure 2). The images in Figure 2 are for 2 wt \% dispersions of MMT and FMT. The MMT image shows a number of aggregates of size exceeding $1 \mu \mathrm{m}$, whereas no such large aggregates are found in the optical micrograph of the FMT (note that the MMT image is darker because the sample transmits less light). The particles in both dispersions were also imaged at higher magnification using TEM (bottom images in Figure 2). In the case of MMT, numerous large clusters are seen, with each cluster evidently comprising many clay platelets. On the other hand, FMT is seen to consist of mostly individual (exfoliated) clay platelets. The images thereby show MMT to consist of a wide distribution of particle sizes, including both nanoscale platelets and their microscale aggregates. By fractionation of MMT, the microscale aggregates are removed and the FMT thus obtained is mostly just the nanoscale fraction.

We also compared the ability of MMT and FMT to gel water. Clays are known for their ability to gel water at high concentrations (8-11). However, their efficiency at gelation varies with the particle size, aspect ratio, polydispersity, and degree of exfoliation. Because gelation is expected to involve a jammed network of particles ("house of cards") $(8,10)$, the most effective gel formation will be by nanoscale platelets because they have the highest aspect ratio. On the other hand, large aggregates such as those seen in MMT (Figure 2) are more globular and thereby of lower aspect ratio; such particles will be inefficient at gelation. Figure 3 shows inverted vials of aqueous samples containing either MMT or FMT. Vial inversion is often used as a simple test of gelation (12). For these experiments, the same amount of each sample was loaded into identical vials, and the vials were kept inverted for several hours. Samples that are stiff gels should be able to hold their weight in the inverted vials (12). From the photograph, the gelation threshold for FMT is at 6 wt \%; i.e., samples with $6 \%$ or higher of FMT hold their weight in the inverted vial. For comparison, the gelation threshold for MMT is $10 \mathrm{wt} \%$, i.e., significantly higher. This shows that FMT is much more efficient at gelling water than MMT, as expected.

One further aspect of interest in Figure 3 is the birefringence of the samples. The samples were photographed 


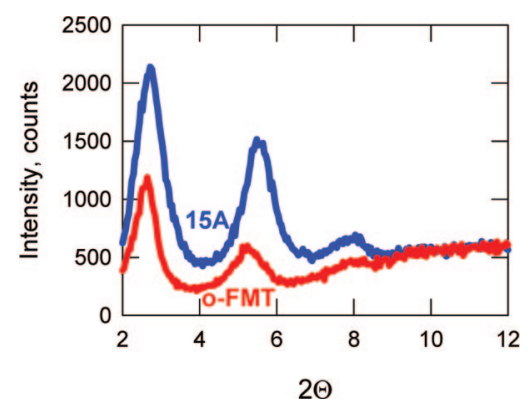

FIGURE 4. XRD spectra for nanocomposites containing $5 \%$ o-FMT or $5 \% 15 \mathrm{~A}$. Both nanocomposites have an intercalated morphology with similar $d$ spacings.

under crossed polarizers to indicate the presence (or absence) of birefringence. While the MMT samples are turbid and thereby transmit less light, they do appear to be isotropic. On the other hand, all three FMT samples show appreciable birefringence, which manifests as a multitude of colors under crossed polarizers. Such birefringence was not seen at low concentrations (up to $4 \mathrm{wt} \%$ FMT) but is visible for $5 \mathrm{wt} \%$ and higher. As is well-known, birefringence is characteristic of anisotropic phases, such as liquid crystals, and its occurrence in particulate dispersions could be due to the alignment of particles (one-dimensional or nematic order) $(11,13)$. In other words, the observations suggest that an isotropic-to-nematic transition occurs in aqueous dispersions of FMT but not MMT. Nematic phase formation by platelike particles has been reported for other clays such as laponite and gibbsite; however, such a phenomenon is precluded if the particles are highly polydisperse $(11,13)$. Thus, the current observations again point to a narrower size distribution of FMT than MMT.

15A/o-FMT Nanocomposites: Morphology. We now describe studies with PS nanocomposites made using o-FMT, i.e., the FMT rendered organophilic by surfactant treatment. As explained earlier, o-FMT is compared with Cloisite 15A (henceforth referred to simply as "15A"), which corresponds to an identical surfactant treatment on the original MMT. Also, from the TGA results (data not shown), the organic content on each clay surface is found to be comparable (60.6 wt \% organics for o-FMT and $61.6 \mathrm{wt} \%$ organics for $15 \mathrm{~A}$ ). Thus, the two organoclays are chemically similar and only differ in terms of their particle size distribution.

As a first step, we characterized the morphology of PS/ 15A and PS/o-FMT nanocomposites using XRD (Figure 4). Both samples show Bragg reflections in their XRD spectra, corresponding to "gallery spacings" within clay stacks of 30 $\AA$ (PS/15A) and $31.5 \AA$ (PS/O-FMT). These values are larger than the gallery spacing of $23.5 \AA$ for the clay powders; the increase in the gallery spacing indicates that the PS chains have intercalated between the platelets $(1-3)$. Thus, both nanocomposites have an intercalated morphology, and the gallery spacing agrees with that found in previous studies of PS/organoclay composites $(14,15)$. Note also that the ratio of the full-width at half-maximum to the peak height is larger for the o-FMT spectrum than for the 15A one. This suggests
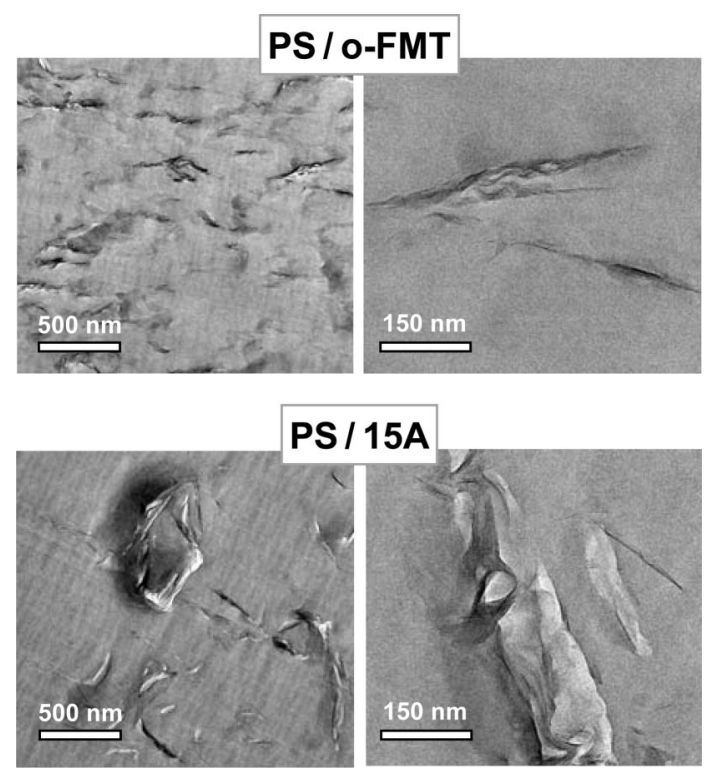

FIGURE 5. TEM images of PS nanocomposites containing $5 \%$ o-FMT (top) or 5\% 15A (bottom). The images on the right are at a higher magnification than those on the left.

that there are fewer platelets in the galleries (stacks) of o-FMT $(14,15)$, as is further corroborated below.

Next, we turn to TEM to directly visualize the morphology of each nanocomposite. Representative images of PS/o-FMT and PS/15A samples, each at $5 \%$ organoclay, are shown in Figure 5. A homogeneous distribution of clay tactoids is seen in the case of the PS/o-FMT composite. The term "tactoid" here refers to a relatively ordered stack of clay platelets $(3,5)$. As seen from the closeup of a single o-FMT tactoid, each tactoid contains a small number of platelets. On the other hand, the PS/15A sample is seen to contain both tactoids as well as larger aggregates ( $\sim \mu \mathrm{m}$ in size). A close up of PS/ $15 \mathrm{~A}$ aggregates reveals that some are tactoids with a larger number of clay platelets. Also, some aggregates do not appear to be ordered stacks but are more isotropic structures; these are possibly clusters of individual tactoids $(3,5)$. The TEM images thus clearly show the superior dispersion of organoclay in the PS/O-FMT composites than in the PS/ $15 \mathrm{~A}$ composites. Although both systems have an intercalated morphology (as per the XRD data), the o-FMT system comes closer to being a nanoscale dispersion of particles, and so the term nanocomposite is more befitting in this case.

15A/o-FMT Nanocomposites: Transparency. Next we evaluate the difference in transparency between the PS/o-FMT and PS/15A composites. The percent transmission is shown as a function of the wavelength for the two systems at various particle loadings in Figure 6. At all wavelengths, the PS/o-FMT composites display higher transmission values. The difference in transmission between the two types of composites is significant: the $5 \%$ PS/o-FMT has roughly the same transmission as the $2 \% \mathrm{PS} / 15 \mathrm{~A}$, and the $10 \%$ PS/O-FMT has roughly the same transmission as the $5 \%$ PS/15A. The inset shows a photograph comparing the two types of composites, and it is evident that the PS/O-FMT sample is more transparent. The increased transparency of PS/o-FMT composites is apparently because large microscale 


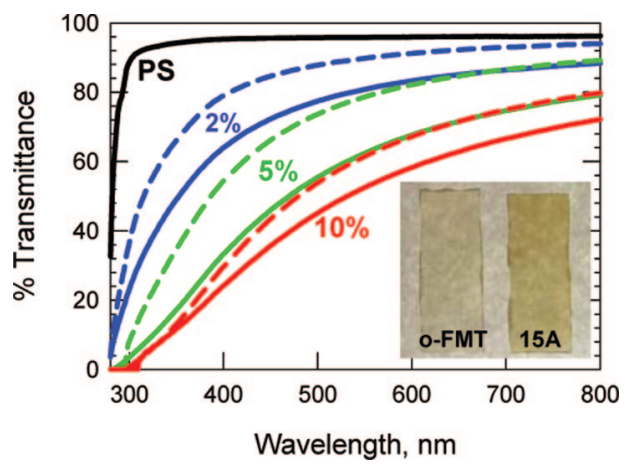

FIGURE 6. Transmission of visible light through $200-\mu \mathrm{m}$-thick nanocomposite films containing either o-FMT (dashed lines) or 15A (solid lines) at different concentrations. The transmission is higher across the wavelength range for the o-FMT samples. This is also evident from the inset photograph comparing $5 \%$ o-FMT and $5 \%$ 15A films.
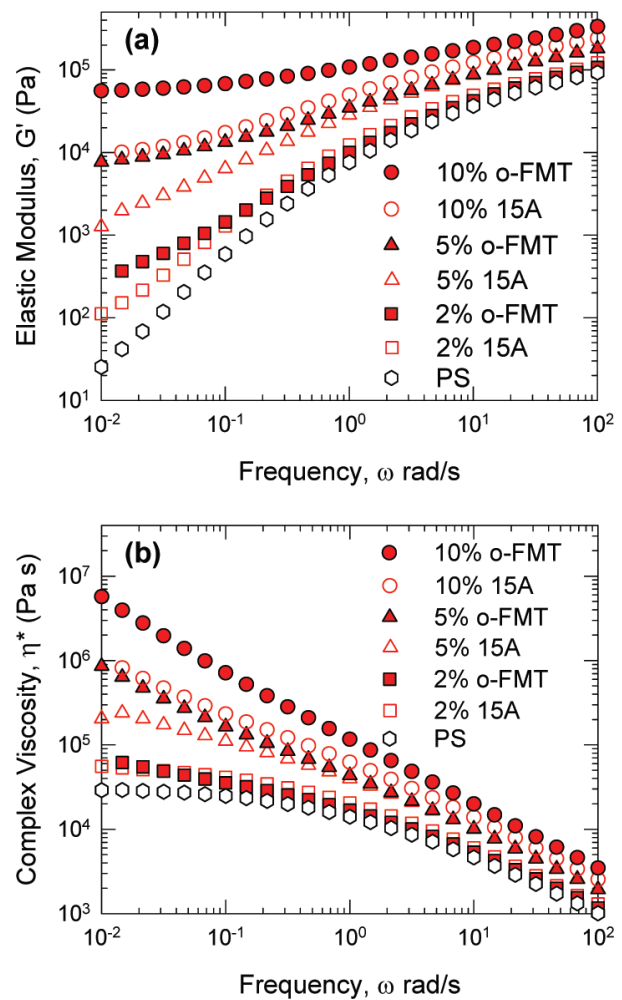

FIGURE 7. Dynamic rheology of PS nanocomposites based on o-FMT and 15A. (a) Data for the elastic modulus $G^{\prime}$. (b) Data for the complex viscosity $\eta^{*}$. Both parameters are significantly higher for the o-FMT samples at equivalent concentrations.

aggregates (that would scatter light and thus contribute to turbidity) are absent, as shown also by the TEM images in Figure 5 .

15A/o-FMT Nanocomposites: Rheology. The differences between o-FMT and 15A are most dramatically seen in the melt rheology of the nanocomposites. Figure $7 \mathrm{a}$ shows the elastic modulus $G^{\prime}$ as a function of the frequency $\omega$ for the parent PS polymer and for the PS/15A and PS/OFMT composites at various clay loadings. Note that $G^{\prime}$ is shown because it is the parameter that most reflects the rheological changes upon clay addition $(1-3)$. The parent polymer exhibits a viscous response with the $G^{\prime}$ scaling at low $\omega$ in a manner characteristic of viscous fluids, i.e., with
$G^{\prime} \sim \omega^{2}(1)$. Adding clay particles causes a systematic increase in $G^{\prime}$, especially at low frequencies, where $G^{\prime}$ becomes less frequency-dependent. At the higher clay loadings, $G^{\prime}$ approaches a low- $\omega$ plateau, which means that the sample relaxes very slowly at long time scales $(1,2)$. Such behavior is characteristic of a volume-filling "jammed" network of particles in the nanocomposite (1).

The significant result from Figure 7 a is that $G^{\prime}$ values for the PS/o-FMT composites are higher than those containing $15 \mathrm{~A}$ at all clay loadings. At a $5 \%$ loading, the $G^{\prime}$ for PS/oFMT at low $\omega$ is larger than that of PS/15A by a factor of 5 , while at a $10 \%$ loading, the difference is a factor of 8 . In effect, $5 \%$ of o-FMT gives a rheological profile similar to that obtained with $10 \%$ of $15 \mathrm{~A}$. These results starkly illustrate the superior efficiency of o-FMT in polymer reinforcement (just as the FMT was more efficient than MMT in gelling water). Note that the differences in $G^{\prime}$ are lowest at the $2 \%$ loading, which is below the percolation (connectivity) threshold. This shows that the greater effectiveness of o-FMT becomes magnified once the particles are connected into a network.

The differences can also be seen in a plot of the complex viscosity $\eta^{*}$ as a function of the frequency $\omega$ in Figure $7 \mathrm{~b}$. As clay is added, $\eta^{*}$ increases at low $\omega$, mirroring the increase in $G^{\prime}$ (1). Again, $\eta^{*}$ is much higher for the PS/o-FMT composites, with the increase being by a factor of 5 at low $\omega$ for the $10 \%$ sample. The rheology data thus indicate that o-FMT is more effective than $15 \mathrm{~A}$ in the formation of jammed networks. Presumably, this difference is because more of the o-FMT particles are participating as cross-links in the network, whereas much of the $15 \mathrm{~A}$ exists in the form of large aggregates and is thereby ineffective at network formation

Finally, we briefly mention our studies to compare the flame retardance of nanocomposites made from o-FMT and $15 \mathrm{~A}$. Both radiant gasification and cone calorimetry experiments were conducted, and the studies are described in the Ph.D. Dissertation of Cipriano (16). The results indicate modest improvements in the flame retardancy of o-FMT nanocomposites compared to those of $15 \mathrm{~A}$.

\section{CONCLUSIONS}

In this paper, we have described a simple method to substantially improve the efficiency of clay particles as a nanoscale filler for polymers. By excluding large particles and aggregates from a commercial MMT sample, we obtained a FMT that, when organically modified (o-FMT), disperses better into PS. The resulting PS/o-FMT nanocomposites were more transparent and displayed $\sim 10 \times$ higher elastic moduli compared to PS nanocomposites prepared with an equivalent commercial organoclay (15A). Such improvements in properties with o-FMT are presumably because the particles are more effective at building a nanoscale connected network.

As a final comment, we should point out that fractionation cannot be done directly on commercial organoclays such as $15 \mathrm{~A}$. To do so, we would first have to disperse the organoclay in a nonpolar organic solvent and then fraction- 
ate the mixture by centrifugation. However, achieving nanoscale dispersion (separation of individual platelets) of organoclays in nonpolar solvents is more difficult compared to dispersing the original hydrophilic MMT into water (7). It is for this reason that we first fractionated MMT and then did an organic modification to obtain o-FMT. We do expect o-FMT to disperse more efficiently in nonpolar solvents than $15 \mathrm{~A}$, and this could be important for the creation of greases and paints at low particle concentration.

Acknowledgment. Funding for this work was provided by the NIST BFRL extramural grants program (Grant 70NANB4H1001). We acknowledge the assistance of UMD undergraduates George Chacko, Gary Cheng, and Alexander Salimian in performing some of the experiments described in this paper.

\section{REFERENCES AND NOTES}

(1) Giannelis, E. P.; Krishnamoorti, R.; Manias, E. Adv. Polym. Sci. 1999, 138, 107.

(2) Alexandre, M.; Dubois, P. Mater. Sci. Eng., R 2000, $28,1$.

(3) Ray, S. S.; Okamoto, M. Prog. Polym. Sci. 2003, 28, 1539.
(4) Chen, B.; Evans, J. R. G.; Greenwell, H. C.; Boulet, P.; Coveney, P. V.; Bowden, A. A.; Whiting, A. Chem. Soc. Rev. 2008, 37, 568.

(5) Paul, D. R.; Robeson, L. M. Polymer 2008, 49, 3187.

(6) Gilman, J. W.; Jackson, C. L.; Morgan, A. B.; Harris, R.; Manias, E.; Giannelis, E. P.; Wuthenow, M.; Hilton, D.; Phillips, S. H. Chem. Mater. 2000, 12, 1866 .

(7) Ho, D. L.; Briber, R. M.; Glinka, C. J. Chem. Mater. 2001, 13, 1923.

(8) van Olphen, H. An Introduction to Clay Colloid Chemistry; Wiley: New York, 1963.

(9) Abend, S.; Lagaly, G. Appl. Clay Sci. 2000, 16, 201

(10) Pignon, F.; Magnin, A.; Piau, J. M.; Cabane, B.; Lindner, P.; Diat, O. Phys. Rev. E 1997, 56, 3281.

(11) Gabriel, J. C. P.; Sanchez, C.; Davidson, P. J. Phys. Chem. 1996, 100,11139

(12) Raghavan, S. R.; Cipriano, B. H. In Molecular Gelators and Their Gels; Weiss, R. G., Terech, P., Eds.; Kluwer Publishers: Amsterdam, The Netherlands, 2004.

(13) Gabriel, J. C. P.; Davidson, P. Adv. Mater. 2000, 12, 9.

(14) Ren, J. X.; Silva, A. S.; Krishnamoorti, R. Macromolecules 2000, 33, 3739.

(15) Krishnamoorti, R.; Ren, J. X.; Silva, A. S. J. Chem. Phys. 2001, 114, 4968.

(16) Cipriano, B. H. Ph.D. Dissertation, University of Maryland, College Park, MD, 2007.

AM8000693 